\title{
Compressive Property of Aluminum Pyramidal Lattice Material
}

\author{
Yunchao Jia ${ }^{1, a}$, Zhidong Guan ${ }^{1, b}$, Xingyi Chen ${ }^{1, c}$ \\ ${ }^{1}$ School of Aeronautic Science and Engineering, Beihang University, Beijing, 100191, China \\ ajiayc630@126.com, bzdguan@buaa.edu.cn, ccarmelo123456@163.com
}

Keywords: Compression; Pyramidal Lattice Material; Finite Element; Plastic

\begin{abstract}
Compressive property of lattice material with pyramidal truss core was investigated under uniform quasi-static compression. Load-displacement response and shape deformations of trusses during compressive loading was simulated by finite element method. The numerical prediction is in good agreement with experimental measurement. Result showed that the compressive process of pyramidal lattice material consist of linear elastic, plastic buckling, secondary contact, softening and densification. Compressive property of lattice material with different truss configuration are compared. Peak compressive stress of lattice material with same relative density depend on the moment of inertia of truss configuration. Truss configuration with higher moment of inertia, can bear higher load.
\end{abstract}

\section{Introduction}

Lattice materials with low-density cores have attracted significant interest owing to their excellent material efficiency and multi-functional application potentials [1-3]. Whilst sandwich panels with honeycomb or aluminum foam cores have already been used in aerospace structures, sandwich panels having metallic lattice truss cores are considered as the most promising new type of advanced lightweight structure to compete with or even replace the honeycomb structures [4].

Most researches on the lattice material with truss core mainly based on the experiments. Cote launched an experimental to explore the in-plane compressive responses and failure mechanisms of pyramidal truss core sandwich columns, the identified failure mechanisms include Euler buckling, shear buckling and face wrinkling [5]. Queheillalt find out that three-dimensional lattice systems have excellent load-bearing ability on account of the stretching-dominated topology structures with high nodal connectivity [6]. Finnegan studied the elastic responses and collapse strengths of the composite cores under out-of-plane compression, indicating that the mechanism of failure is not activated until delamination is suppressed [7]. Fan observed the mechanical behaviors of the sandwich panels by out-of-plane compression, in-plane compression and three-point bending. Pointed out the imperfections of lattice materials, such as the waviness of the struts, non-circular cross sections and cantilever ribs, greatly influenced their performance [8]. Feng carried out an experiment to examine the mechanical response of pyramidal truss core sandwich columns [9]. Wang investigated the mechanical properties under out-of-plane compression and shear loading. Results showed that the mechanical behavior of the pyramidal lattice truss core sandwich panels depends on the relative density of core and the material properties of truss members [10].

Up to now, as far as we know, only few numerical investigations have been done on the compressive property of lattice material. In the present work, we studied the mechanical response and shape deformation of aluminum lattice material with pyramidal truss cores under uniform quasi-static compressive loading by finite element software ABAQUS, as an initial step in understanding the performance of this promising material. The difference between experiment and numerical prediction is compared. Besides, we compare its compressive property with different truss configurations. 


\section{Experiment}

The experiment in this paper is cited from reference [9]. Aluminum lattice material was tested in through-thickness compression following the ASTM C365 guideline. The test was carried out at ambient temperature and loading rate of $1 \mathrm{~mm} / \mathrm{min}$. The specimen is shown in Fig. 1.

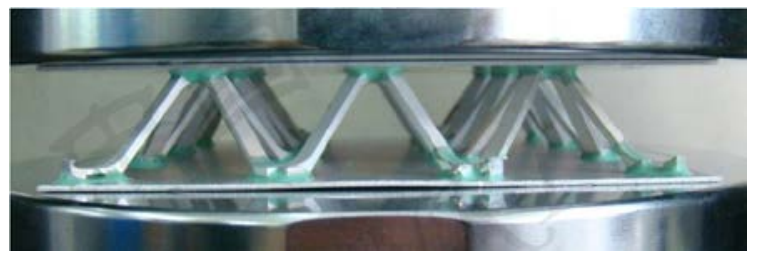

Fig. 1 Specimen

\section{Finite element model}

To further explore the compressive response of pyramidal lattice material, the method of finite element is employed. Fig.2 shows the model set up in the commercial finite element code ABAQUS. The simulated result is shown in Fig. 3

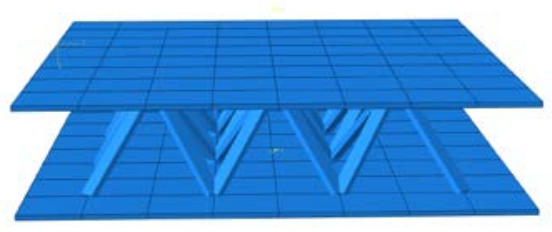

Fig. 2 Finite element model

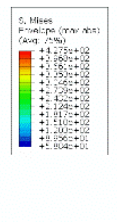

Fig. 3 Simulated result

The geometrical dimensions of the simulated lattice material were identical to those used in experimental measurements. The panel size is $100 \mathrm{~mm} \times 100 \mathrm{~mm} \times 0.77 \mathrm{~mm}$ and the core size is $86.64 \mathrm{~mm} \times 86.64 \mathrm{~mm} \times 17 \mathrm{~mm}$. The truss size is $19.87 \mathrm{~mm} \times 3.2 \mathrm{~mm} \times 1.34 \mathrm{~mm}$ with the tilt angle of $49^{\circ}$, resulting in a relative density of $3.75 \%$.

The panel was set as rigid body and the trusses were built as beam with the element type of B31. The panel and truss were made of aluminum whose material property and plastic property are listed in Table 1 and Table 2. The bottom panel was fixed to simulate the experimental support condition and a reference point was defined to couple with the upper panel. The compressive load was applied on the reference point.

\begin{tabular}{cc}
\multicolumn{2}{c}{ Table 1 Material property of aluminum } \\
\hline Parameters & Value \\
\hline Elastic modulus [Gpa] & 70 \\
Poisson's ratio & 0.33 \\
Density $\left[\mathrm{Kg} / \mathrm{m}^{3}\right]$ & 2700 \\
\hline
\end{tabular}

Table 2 Plastic property of aluminum

\begin{tabular}{ccccccccc}
\hline Ture stress [Mpa] & 243.85 & 288.44 & 297.41 & 305.23 & 311.9 & 317.36 & 321.61 & 324.09 \\
\hline Plastic strain & 0 & 0.0091 & 0.0194 & 0.0286 & 0.0374 & 0.0462 & 0.0548 & 0.0613 \\
\hline
\end{tabular}

\section{Results and discussion}

The load versus displacement curves of FE analysis and experiment under through thickness compression are plotted in Fig. 4. The simulated peak compressive stress and the corresponding experimental result are listed in Table 3. From Fig. 4 and Table 3, we can see that the simulated result agree well with the measured one. The over-prediction peak load for the pyramidal lattice material is attributed to the presence of imperfection in the manufacturing process, which was not involved in the FE simulation. When the distorted truss members contacted the panel, the load rose up and 
reached the second peak. The second peak load value of experiment is higher than the FE analysis one due to the strengthen ends of trusses in the manufacturing process, which alleviate the distortion of trusses.

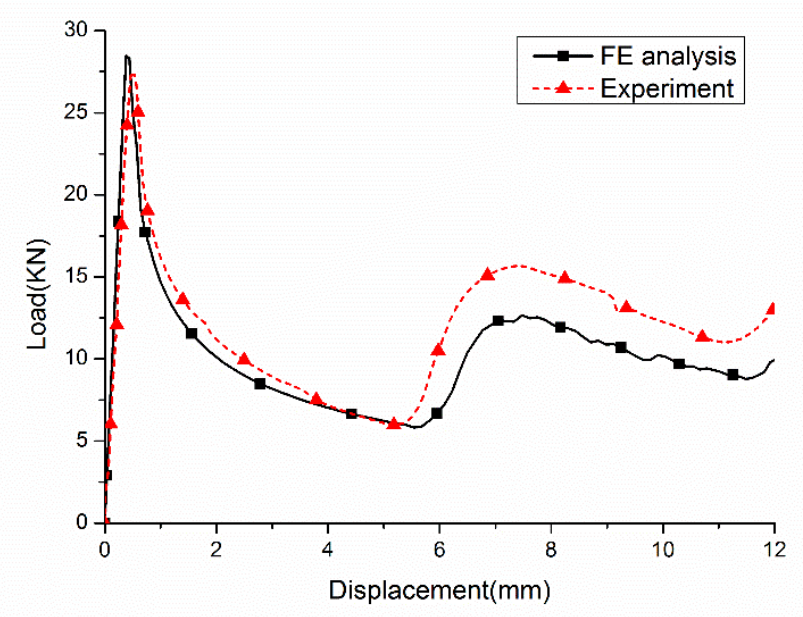

Fig. 4 The comparison of load-displacement curves between FE analysis and experiment

Table 3 Comparison of peak compressive stresses $\left(\sigma_{\mathrm{pk}}\right)$ between FE analysis and experiment

\begin{tabular}{cccc}
\hline & FE analysis & Experiment & Error (\%) \\
\hline$\sigma_{\mathrm{pk}}[\mathrm{Mpa}]$ & 3.79 & 3.65 & 3.84 \\
\hline
\end{tabular}

A representative lattice cell contains four trusses. There are two kinds of typical deformation mode. The shape deformations of the trusses, ascertained by experiment, are presented in Table 4. For deformation mode 1 , some of the trusses bend outwards and the other trusses bend inwards, while all the trusses bend inwards for deformation mode 2. Deformation mode 1 took place for the trusses close to the edge and mode 2 happened for the trusses in the middle of the specimen.

Table 4 Shape deformations of the trusses during compressive loading (displacement $=\mathrm{u}$ )

\begin{tabular}{|c|c|c|c|c|}
\hline & \multicolumn{2}{|c|}{ Deformation mode 1} & \multicolumn{2}{|c|}{ Deformation mode 2} \\
\hline & Experiment & FE analysis & Experiment & FE analysis \\
\hline $\begin{array}{l}\text { Linear elastic } \\
(\mathrm{u}=0.22 \mathrm{~mm})\end{array}$ & & & & \\
\hline $\begin{array}{c}\text { Peak load } \\
(\mathrm{u}=0.39 \mathrm{~mm})\end{array}$ & & & & \\
\hline $\begin{array}{c}\text { Plastic } \\
\text { buckling } \\
(\mathrm{u}=2.06 \mathrm{~mm})\end{array}$ & & & & \\
\hline $\begin{array}{c}\text { Secondary } \\
\text { peak load } \\
(\mathrm{u}=7.75 \mathrm{~mm})\end{array}$ & & & & \\
\hline $\begin{array}{l}\text { Densification } \\
(\mathrm{u}=11.59 \mathrm{~mm})\end{array}$ & & & & \\
\hline
\end{tabular}


Inspection of the figures indicates that the trends in the simulation are similar to those ascertained by experiment. From Table 4 and Fig. 4, it can be clearly seen that the compression process consist of five stages: linear elastic, plastic buckling, secondary contact, softening and densification.

Linear elastic. Firstly, the load increased in proportion to displacement. Initial linear responses were observed for all the trusses. As the load increased, individual truss member buckling was observed. And all the trusses buckled quickly at the end of this stage due to the equally applied compressive load. At the peak compressive load, it can be seen that all truss members had fully developed plastic hinges at mid-truss locations according to the FE analysis result.

Plastic buckling. As deformation progressed, the truss members continued to buckle and the trusses began to bend outwards or inwards as mentioned before in Table 4 . Then the lattice material lost its ability to carry load, leading to a rapid load drop.

Secondary contact. When the displacement reached $5.67 \mathrm{~mm}$, the plastic hinges impinged on the face-sheets and the core strength increased once more due to the effective shortening of the truss members, similarly to the linear elastic stage. The load kept increasing until the remaining effective trusses buckled again, where the material reached the secondary peak load.

Softening. Core softening was observed once the peak compressive strength of the material was surpassed. Coinciding with the first stage, initiation of buckling of the remaining effective lattice truss members and the formation of plastic hinges near the center of the truss members occurred.

Densification. Continued loading resulted in a large displacement $(\mathrm{u} \geq 11.59 \mathrm{~mm})$, at which point the load-carrying capacity increased rapidly as most parts of the deformed trusses made contact with the face sheets. Initial contact between the core members and the faces was restricted in extent, resulting in only a small load elevation, as shown in Fig. 4. It can be predicted that further deformation will induce the extensive contact that causes rapid densification evidently.

\section{Comparison of different truss configurations}

Based on the aforementioned FE model, we compared the compressive properties of different truss configurations. Five types of configuration were selected, including rectangle, circle, square, box and pipe, as shown in Fig. 5.

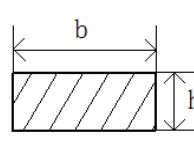

rectangle

b= 3.20

$\mathrm{h}=1.34$

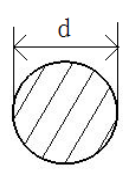

circle $\mathrm{d}=2.34$
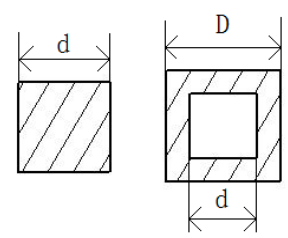

box

$\mathrm{D}=5.11$

$\mathrm{d}=3.00$

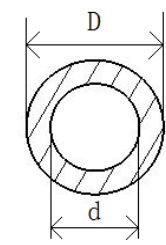

pipe

$\mathrm{D}=3.08$

$\mathrm{d}=2.00$

Fig. 5 Five types of truss configuration

To ensure the result are valid, all the truss configurations have the same cross-sectional area, which means the lattice material with different configurations have the same relative density. The simulated peak compressive stresses of different truss configurations are listed in Table 5

\begin{tabular}{cccccc} 
Table 5 Peak compressive stresses & $\left(\sigma_{\mathrm{pk}}\right)$ of different truss configurations \\
\hline Configuration & rectangle & circle & square & box & pipe \\
\hline$\sigma_{\mathrm{pk}}[\mathrm{Mpa}]$ & 3.79 & 3.91 & 4.16 & 4.43 & 4.62 \\
\hline
\end{tabular}

The compressive strength of hollow trusses, including box and pipe, were higher than that of solid trusses, consisting of rectangle, circle and square, with the same relative density. The increased strength resulted from an increase in the buckling resistance of hollow trusses because of their higher moment of inertia. Moment of inertia of different truss configurations are listed in Table 6. 
Table 6 Moment of inertia of different truss configurations

\begin{tabular}{cccccc}
\hline Configuration & rectangle & circle & square & box & pipe \\
\hline Moment of inertia, I $\left[\mathrm{mm}^{4}\right]$ & 0.642 & 1.461 & 1.530 & 3.093 & 3.607 \\
\hline
\end{tabular}

Peak compressive stresses of lattice material with same relative density depend on the moment of inertia of truss configurations. Generally, truss configuration with higher moment of inertia, can bear higher load.

\section{Conclusion}

The compressive property of aluminum lattice material with pyramidal truss core was investigated under uniform quasi-static compression. Load-displacement response was measured by finite element software ABAQUS, with good agreement in numerical predictions and experimental measurements.

The result exhibited characteristics typical of aluminum lattice material including linear elastic response, plastic buckling, secondary contact followed by softening and finally densification. Throughout the compression process, there are two similar load rising and falling sections. The first section was caused by elastic response and trusses buckling due to plastic hinges developed at mid-truss locations, while the second section took place because of the contact between trusses and panels and plastic buckling developed in the remaining effective truss members.

Compressive property of lattice material with different truss configurations are compared. Peak compressive stress of lattice material with same relative density depend on the moment of inertia of truss configuration. Truss configuration with higher moment of inertia, can bear higher load.

\section{References}

[1] Evans AG, Hutchinson JW, Fleck NA, et al. The topological design of multifunctional cellular metals. Progress in Materials Science. 2001, 46(3-4):309-327.

[2] Wadley HNG. Multifunctional periodic cellular metals. Philosophical Transactions of the Royal Society a-Mathematical Physical and Engineering Sciences. 2006, 364(1838):31-68.

[3] Wang B, Wu LZ, Jin X, et al. Experimental investigation of 3D sandwich structure with core reinforced by composite columns. Materials \& Design. 2010, 31(1):158-165.

[4] Zhang QC, Lu TJ. Experimental and simulated compressive properties of work-hardened X-type lattice truss structures. Acta Mechanica Solida Sinica. 2012, 25(2):111-116.

[5] Cote F, Biagi R, Smith HB, et al. Structural response of pyramidal core sandwich columns. Int. J. Solids Struct. 2007, 44:3533-3556.

[6] Queheillalt, DT, Murty Y, Wadley HNG. Mechanical properties of an extruded pyramidal lattice truss sandwich structure. Scripta Mater. 2008, 58:76-79.

[7] Finnegan K, Kooistra G, Wadley HNG, et al. The compressive response of carbon fiber composite pyramidal truss sandwich cores. Int. J. Mater. Res. 2007, 98:1264-1272.

[8] Fan HL, Meng FH, Yang W. Mechanical behaviors and bending effects of carbon fiber reinforced lattice materials. Arch. Appl. Mech. 2006, 75:635-647.

[9] Feng LJ. Fabrication and mechanical analysis of aluminum pyramidal truss sandwich panels. Harbin Institute of Technology. 2012

[10] Wang B, Wu LZ, Ma L, Sun YG, Du SY. Mechanical behavior of the sandwich structures with carbon fiber-reinforced pyramidal lattice truss core. Mater. Des. 2010, 31:2659-2663. 